Errata

\title{
Smooth Non-Bernoulli $K$-Automorphisms
}

\author{
A. Katok \\ Department of Mathematics, University of Maryland, College Park, MD, USA
}

Invent. math. 61, 291-299 (1980)

The note added in the proof on page 299 does not refer to the paper of A. Katok but to the preprint by $\mathrm{M}$. Brin, number 9 in the list of references. The asterisks were deleted by mistake.

\section{Positivity Notions for Coherent Sheaves over Compact Complex Spaces}

\author{
Joshua H. Rabinowitz \\ Department of Mathematics, University of Illinois at Chicago Circle, Chicago, IL 60680, USA
}

Invent. math. 62, 79-87 (1980)

The Corollary to Theorem 2 on page 81 should read

Let $X$ be a normal irreducible compact complex space, $\mathscr{P} \rightarrow X$ a primary positive coherent sheaf of generic fiber dimension one. Then $H^{k}(X, \mathscr{S} \otimes \mathscr{K})=0$ for all $k \geqq 1$, where $\mathscr{K}$ is the "canonical sheaf" of $X$. 